\title{
Observation of Soils: From the Field to the Microscope
}

\section{File 1: The Multiscalar Nature of Soils}

As emphasized by W. Kubiëna, “... there exists no other method capable of revealing the nature and complexity of soil polygenesis in so much detail as thin-section micromorphology and at the same time enabling one to follow and explain its formation...". This sentence, cited by Fedoroff (1971), highlights the aim of soil micromorphology: looking at a soil from the inside and at various scales, from the optical microscope to synchrotron imaging. Soils constitute multiscalar objects by definition, from their soilscape (at the landscape scale), to their profile and its horizons to the atomic interactions between the smallest minerals and organic molecules. Micromorphology enters the soil investigations at the multi-centimetre scale (see "File 3") at which the thin section is made. The scales of observation span from the millimetre to the micrometre, and even down to the nanometre using electron microscopy (see "File 7" and "File 8"). Therefore, the micromorphological approach is based on multiscalar observations because the different features and properties of soils require different magnifications; in addition, this approach is twofold using composition and fabric (see "File 9").

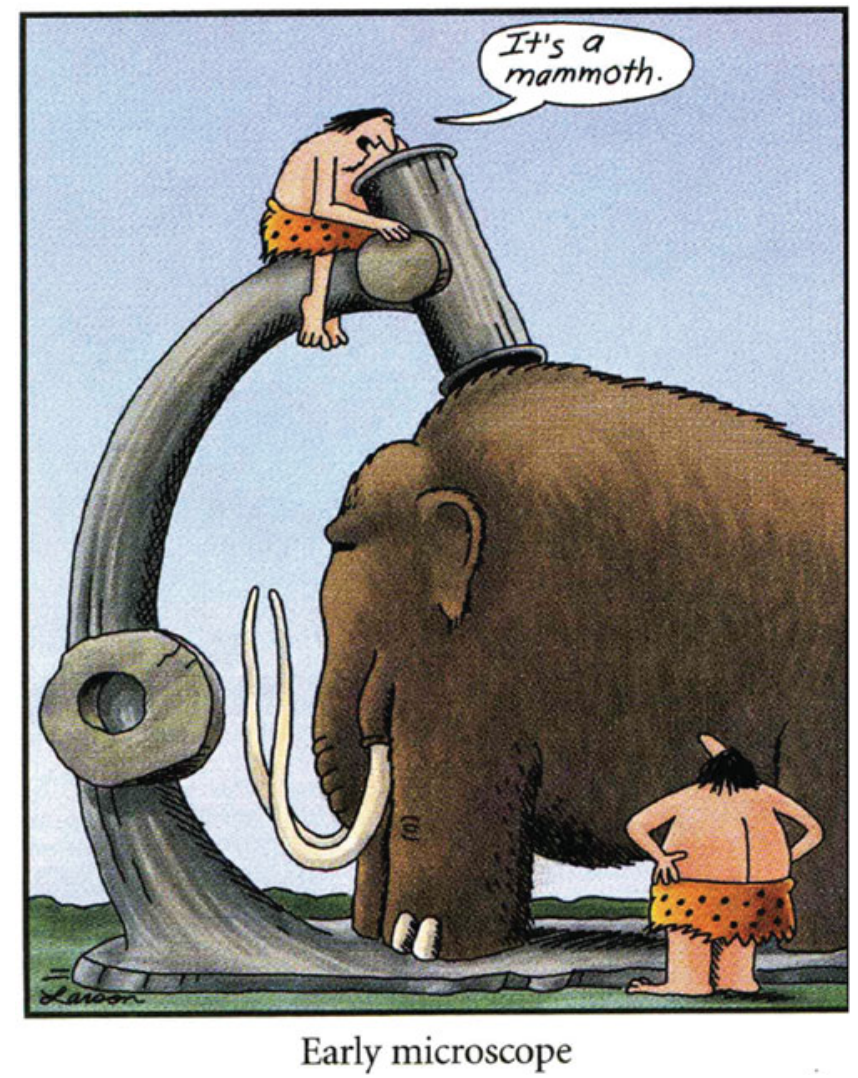

Cartoon from the Larsen's Far Side. This cartoon has been published in the Proceedings of the International Working Meeting on Soil Micromorphology in San Antonio, Texas, July 1988. 

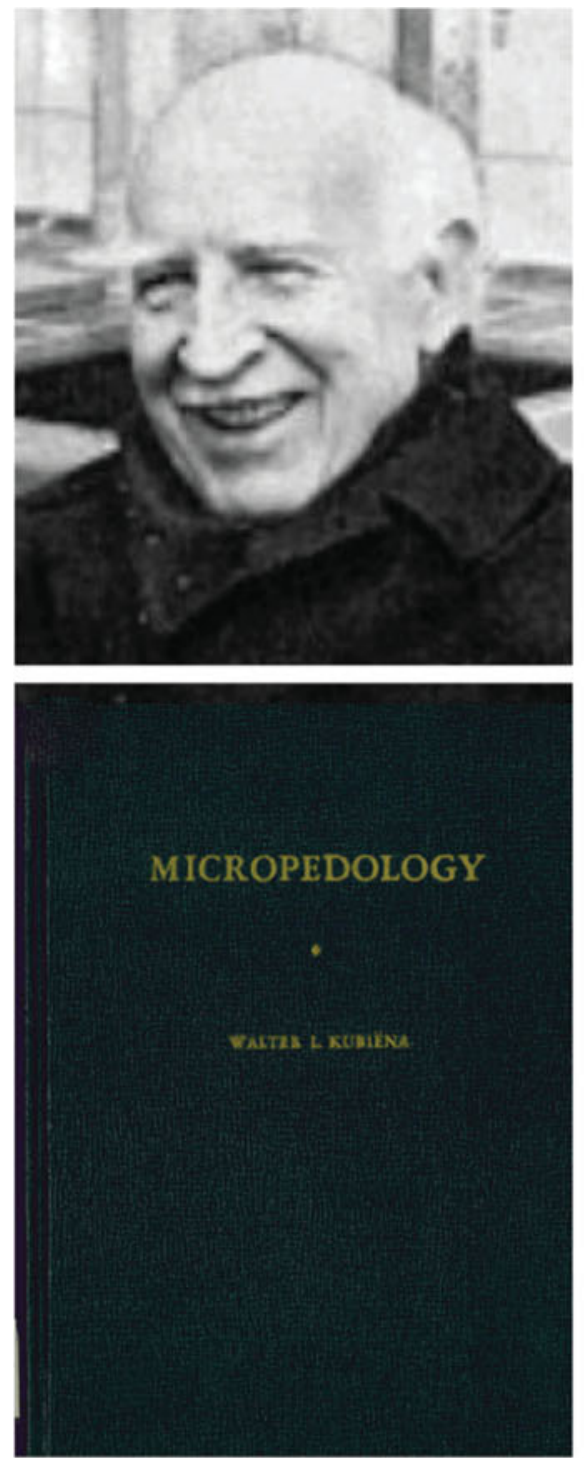

HANDBOOK for

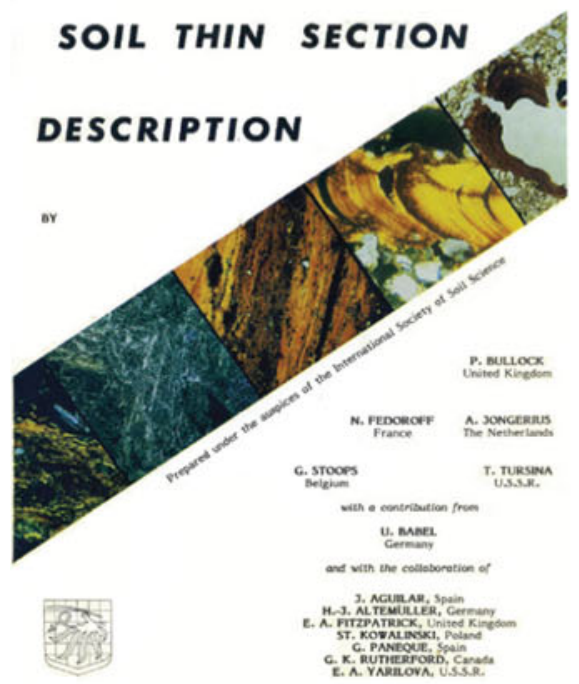

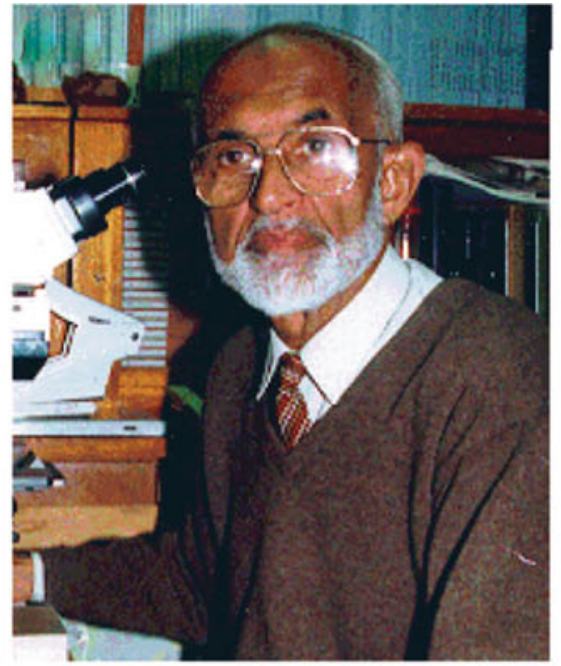

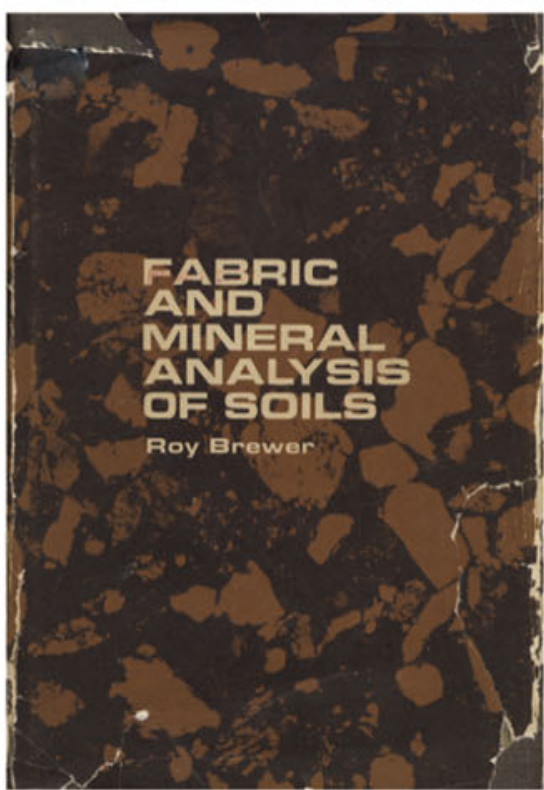

Micromorphology of Soils

E.A. FitzPatrick
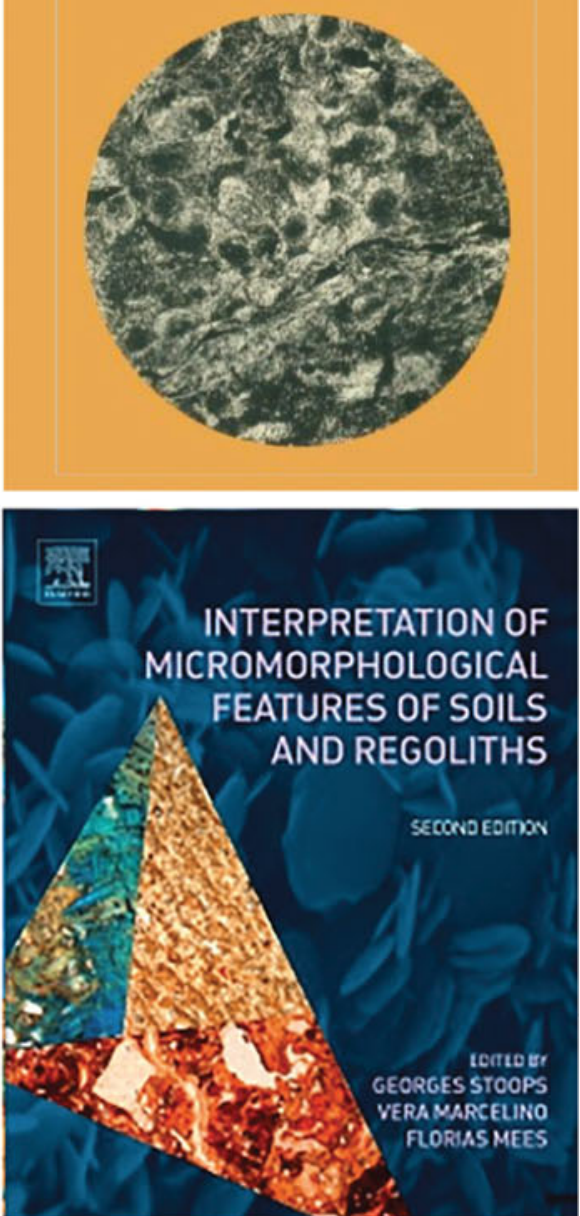


\section{File 2: History of Micromorphology}

Soil micromorphology is a relatively recent method, first popularized by a book by Kubiëna (1938). This method revolutionized the way that a soil was observed because it was studied from the inside, i.e. the inner organization. Indeed, during the "Symposium on the Age of Parent Material and Soils", in 1970, Walter Kubiena, the father of the micropedology, stated: “... there exists no other method capable of revealing the nature and complexity of soil polygenesis in so much detail as thin-section micromorphology and at the same time enabling one to follow and explain its formation ..." (Fedoroff 1971).

\section{Captions from upper left corner to lower right corner.}

1. Photograph of Walter L.K.R. von Kubiëna (1897-1970): considered as the father of soil micromorphology, W. Kubiëna investigated microscopic crystal formation and neoformation in soils during his several stays in the USA (mostly during 1931 and 1933). The method he applied is based on thin sections and microscopic observation with a polarizing microscope, which provided him with new insights into the variety of forms encountered in soils. During the next few decades, he made soil micromorphology his main field of research. Invited to give lectures at Iowa State College in Ames (USA), he decided to gather his experience into a book, Micropedology (Kubiëna 1938), which became the first international standard work in soil micromorphology. (Photo credit: www.spektrum.de)

2. Photograph of Ewart A. FitzPatrick (1926-2018): author of one of the most successful books in soil micromorphology published in 1984, and simply titled Micromorphology of Soils (FitzPatrick 1984), the work by E. A. FitzPatrick is renown for its clarity, magnificent illustrations, and its simple and useroriented approach, avoiding complex vocabulary. E. A. FitzPatrick also developed techniques and protocols optimising the fabrication and interpretations of thin sections. He received the Kubiëna Medal of the International Union of Soil Sciences in 1996. (Photo credit: International Union of Soil Sciences)

3. Photograph of Georges Stoops: as a major promoter of soil micromorphology during the last 40 years, G. Stoops fundamentally contributed to the standardization of the vocabulary used in thin section description, as well as to the classification and interpretation of micromorphological features (Stoops 2003). He received the Kubiëna Medal of the International Union of Soil Sciences in 1992.

4.-9. Covers of the main milestone books in soil micromorphology in a chronological order: Kubiëna (1938), Brewer (1964), FitzPatrick (1984), Bullock et al. (1985), Stoops (2003), and Stoops et al. (2018). 


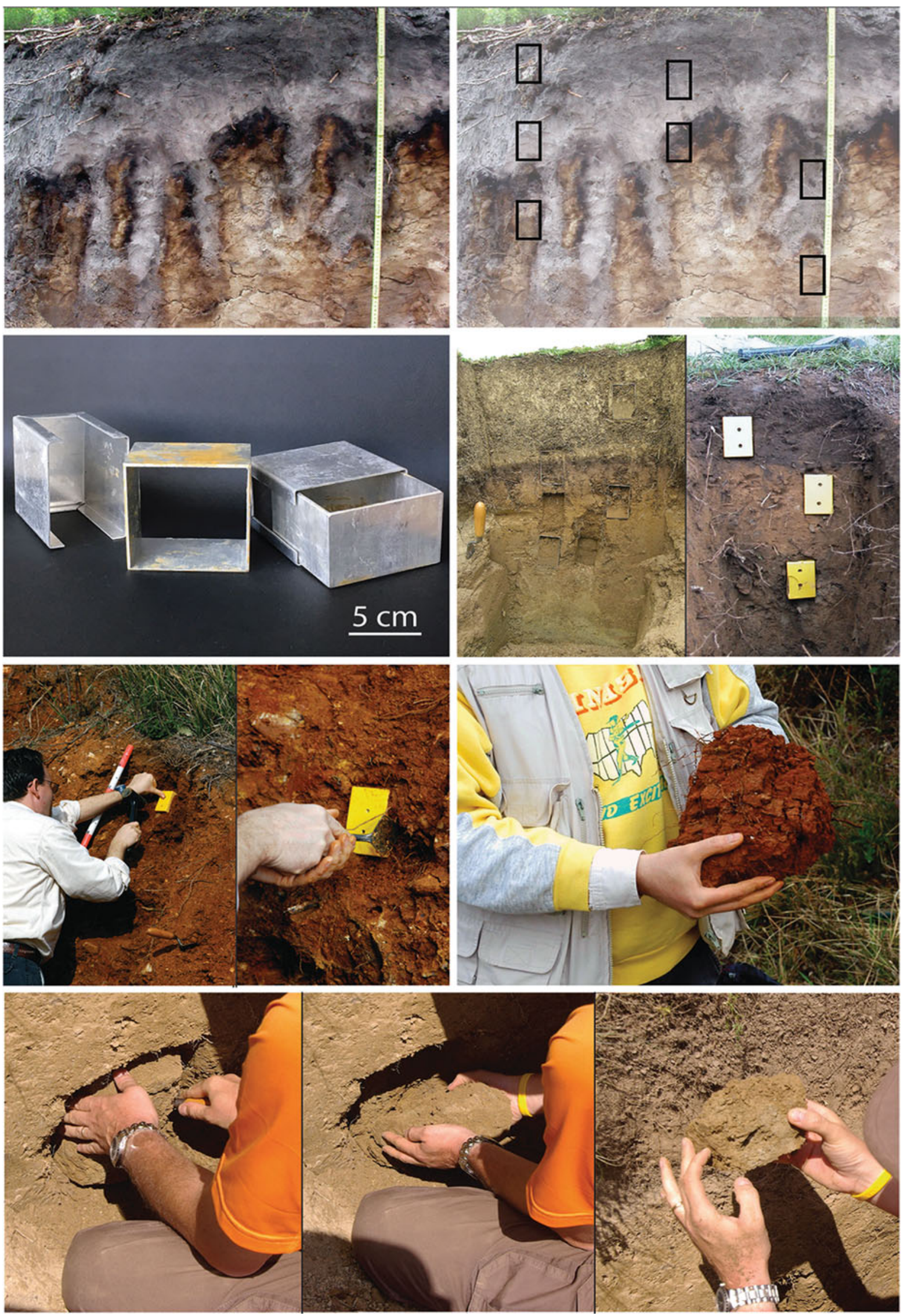




\section{File 3: Observation and Sampling of Soils}

There are various ways to sample soil profiles in the field to get the right soil portion of interest that represents the horizon variability. Sampling can focus on horizons, transitions, or specific soil features. The most conventional sample selection is based on the use of Kubiëna boxes of various shapes, sizes, and compositions. They are mainly used in soils with fine textures. However, the soil texture can sometimes be an obstacle to conventional field sampling: a manual block extraction sampling technique is then performed in order to get enough soil material for further impregnation in the laboratory. Moreover, a plaster-impregnated patch of burlap can be used to encase and sample a large block of undisturbed soil (Goldberg and Macphail 2003).

\section{Captions from upper left corner to lower right corner.}

1. Profile of a podzol from Fontainebleau Forest (Paris Basin, France) with its six horizons (O, A, E, Bh, Bs, C). The E horizon's lower boundary has deep vertical tongues. Below the easily observed E and Bh horizons, there are bands enriched in iron, corresponding to the diffusion of organometallic complexes following grain-size irregularities of the parent material. Scale $=80 \mathrm{~cm}$.

2. Examples of sampling locations on the profile described above. The three rectangles on the left side indicate the sampling location of each horizon. The two boxes in the middle show the horizon's transitions. Finally, the right side boxes point out special features, i.e. tongues and mottles of organometallic complexes.

3. Homemade Kubiëna boxes ready to collect undisturbed samples: these are empty solid square $(8 \times 8 \mathrm{~cm})$ aluminium frames with sharp edges. Lids are used to preserve the undisturbed soil after sampling. An arrow must be drawn on the box pointing to the top (i.e. the ground's surface). A sample number can also be written directly on the box's lid with a felt pen. Such boxes can be easily cleaned with water and ethanol and reused.

4. Homemade Kubiëna boxes of different materials inserted directly in a soil profile. Left: metal boxes in a loamy soil; right: plastic boxes in a silty soil.

5. Left: insertion of a plastic Kubiëna box in a soil profile with the help of a rubber hammer. Right: extraction of the box with the help of a trowel (a knife can equally be used); after having clearly delimited the border of the box, it is gently extracted to avoid any disturbance.

6. Sampling of a large soil block without a field Kubiëna box: such samples must be wrapped with appropriate protection; back in the laboratory, they will be directly impregnated in a tray.

7. From left to right: example of the extraction of a block from a loamy soil profile with a trowel. 

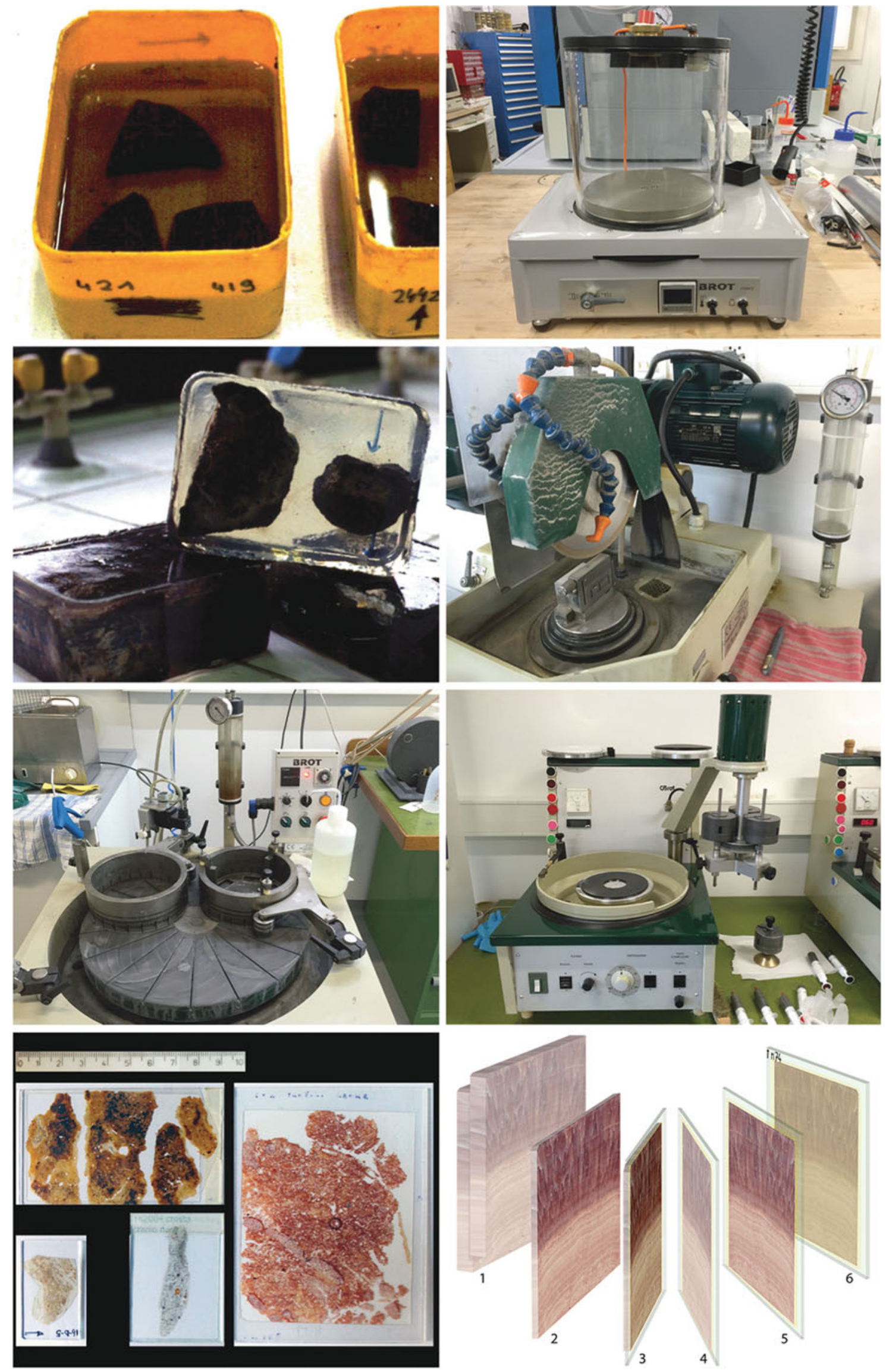


\section{File 4: How to Make Thin Sections}

The micromorphological observation of soils starts with the fabrication of thin sections. This process is generally long because soil samples must be impregnated to solidify them: a polystyrene-based resin, often with a density close to water, is poured on samples. With time, it will penetrate inside the soil pores and harden. Making thin sections requires some specific equipment. The series of photographs included here provides only the major steps involved in thin section preparation. For more information see Murphy (1986) and Benyarku and Stoops (2005).

\section{Captions from upper left corner to lower right corner.}

1. Impregnation of soil blocks inside trays filled with polystyrene-based resin with an added accelerator and a catalyst (laboratory at the University of Ghent, Belgium). Acetone can also be used as a diluent, if needed.

2. Vacuum system used to remove residual air from impregnated samples at the University of Lausanne, Switzerland. This process is often used when the polystyrene-based resin is still liquid, as vacuum can help to access micropores and partly avoid problems related to air bubbles.

3. Impregnated and hard samples ready for sawing (laboratory at the University of Ghent, Belgium). The arrow shows the top of the soil.

4. Diamond saw machine used to prepare slabs for grinding, polishing, and lapping at the University of Lausanne, Switzerland. The aim is to obtain slabs slightly smaller than the size of the glass slide chosen to mount the thin section, and of a thickness depending on the type of saw, but generally about $1-2 \mathrm{~cm}$.

5. Lapping machine used to flatten the slabs and frost the glass slide (laboratory at the University of Lausanne, Switzerland).

6. Polishing machine for ultra-smooth surfaces (laboratory at the University of Lausanne, Switzerland). For example, such thin sections are used for cathodoluminescence (see "File 6") or microprobe chemical imaging (see "File 7").

7. Examples of various sizes of soil thin sections. The most common sizes are the following: standard $(28 \times$ $48 \mathrm{~mm})$, medium $(60 \times 90 \mathrm{~mm})$, large $(90 \times 120 \mathrm{~mm})$, mammoth $(120 \times 180 \mathrm{~mm})$.

8. Example of the succession of phases needed to prepare soil thin sections: (1) slab extract from impregnated blocks using diamond saw machines; (2) first face lapping; (3) bonding of lapped face to frosted glass slide; (4) thinning of the bulk face; (5) grinding and lapping of the thin section; (6) thin section polishing; after this phase, the thin section is $30 \mu \mathrm{m}$ thick and ready for observation. Thin sections can be glass-covered or not. Uncovered thin sections are usually used for staining, selective extraction (see "File 8"), cathodoluminescence (see "File 6"), backscattered electron or chemical imaging (see "File 7", "File 77", and "File 78"). 

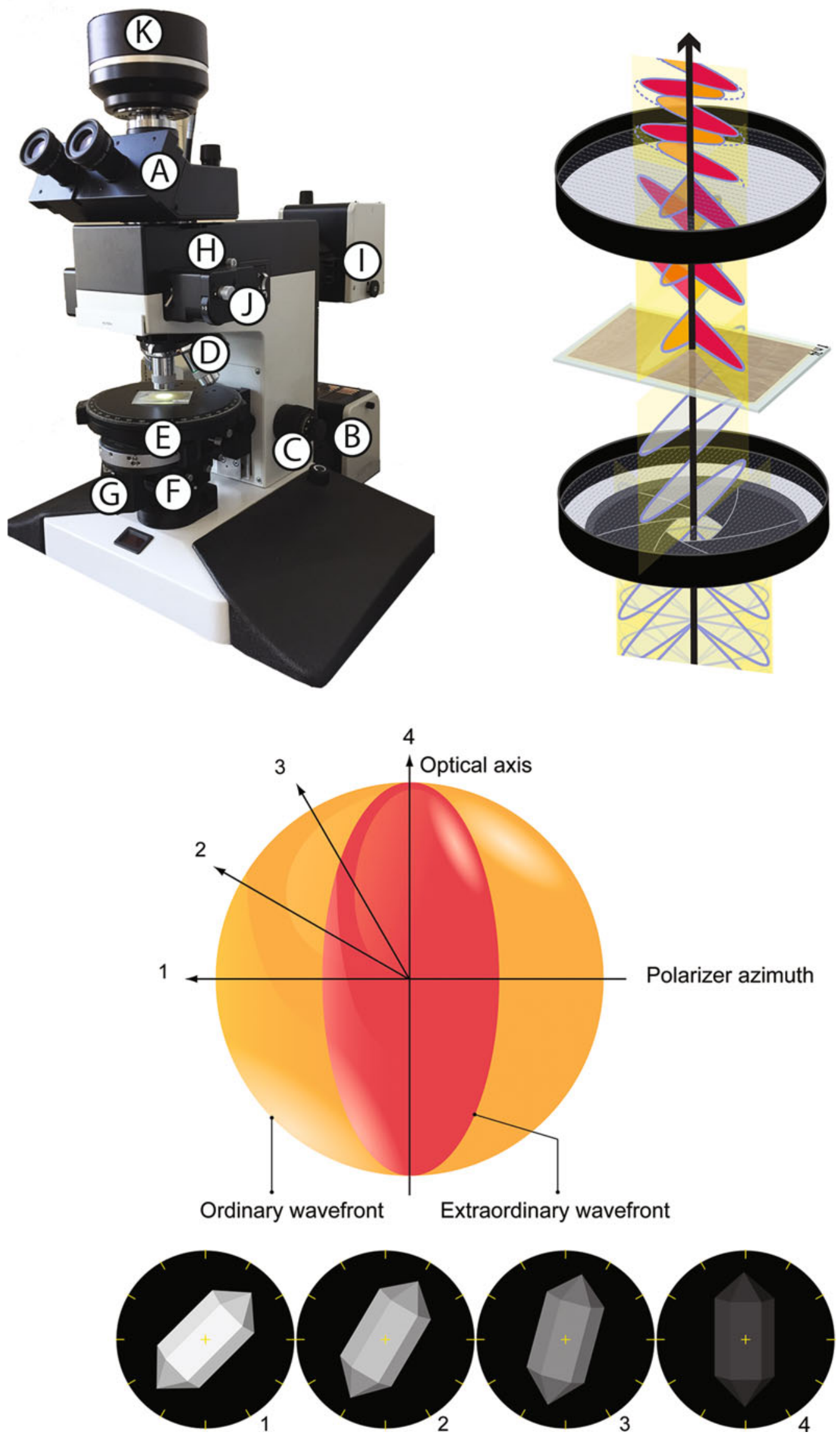


\section{File 5: The Polarized Light Microscope}

In order to perform micromorphological observations, the polarized light microscope is the most appropriate tool. It couples the magnification of a conventional optical microscope with light polarization induced by a polarizer and an analyser located along the optical light pathway. Micromorphologists use the optical anisotropic properties of soil constituents for their identification and the observation of their potential transformation.

\section{Captions from upper left corner to lower right corner.}

1. An example of a fully equipped polarized light microscope with fluorescence: (A) binocular observation tubes (eyepieces), (B) source of transmitted light, (C) focus, (D) revolving objectives, (E) circular rotating stage, (F) $360^{\circ}$ rotating polarizer, $(\mathrm{G})$ condenser, $(\mathrm{H})$ analyser slide, (I) source of incident light (used for fluorescence), (J) fluorescence filters, (K) microphoto or video camera.

2. Principle of light polarization with two polarizing filters: first, the light comes from a white source at the bottom of the sketch. There are two polarizing filters along the optical pathway, the polarizer and the analyser. The polarizer is below the slide stage and fixes the vibration direction of light in a single direction, let us say north-south. The analyser allows vibration of the light perpendicular to the polarizer direction (i.e. eastwest). The analyser position is above the objectives and can be slid in and out. When the analyser is in, this configuration is called cross-polarized light, with no light passing through the system (the view field is totally dark): this is abbreviated as XPL (cross-polarized light). If an anisotropic soil thin section is placed between the two polarizers, polarization colours appear due to different refractive indices (refringence) of objects in the soil. If the analyser is out, this configuration is abbreviated as PPL (plane-polarized light). The degree to which edges and surface imperfections of crystals is visible in PPL is called "relief". Moreover, linear traces can sometimes be observed through a mineral section in PPL or XPL: they are cleavage traces. Their angular relationships are used to identify some minerals.

3. Principle of mineral extinction: the intensity of the polarization colours varies during rotation of the stage from maximum brightness (1) to zero (extinction; 4). This succession is cyclic when rotating the stage $360^{\circ}$. Whenever the specimen is in extinction, the permitted vibration directions of light passing through are parallel with those of either the polarizer or analyser. However, if the thin section material is isotropic, it remains totally dark (extinction) when the stage is rotated through $360^{\circ}$. 

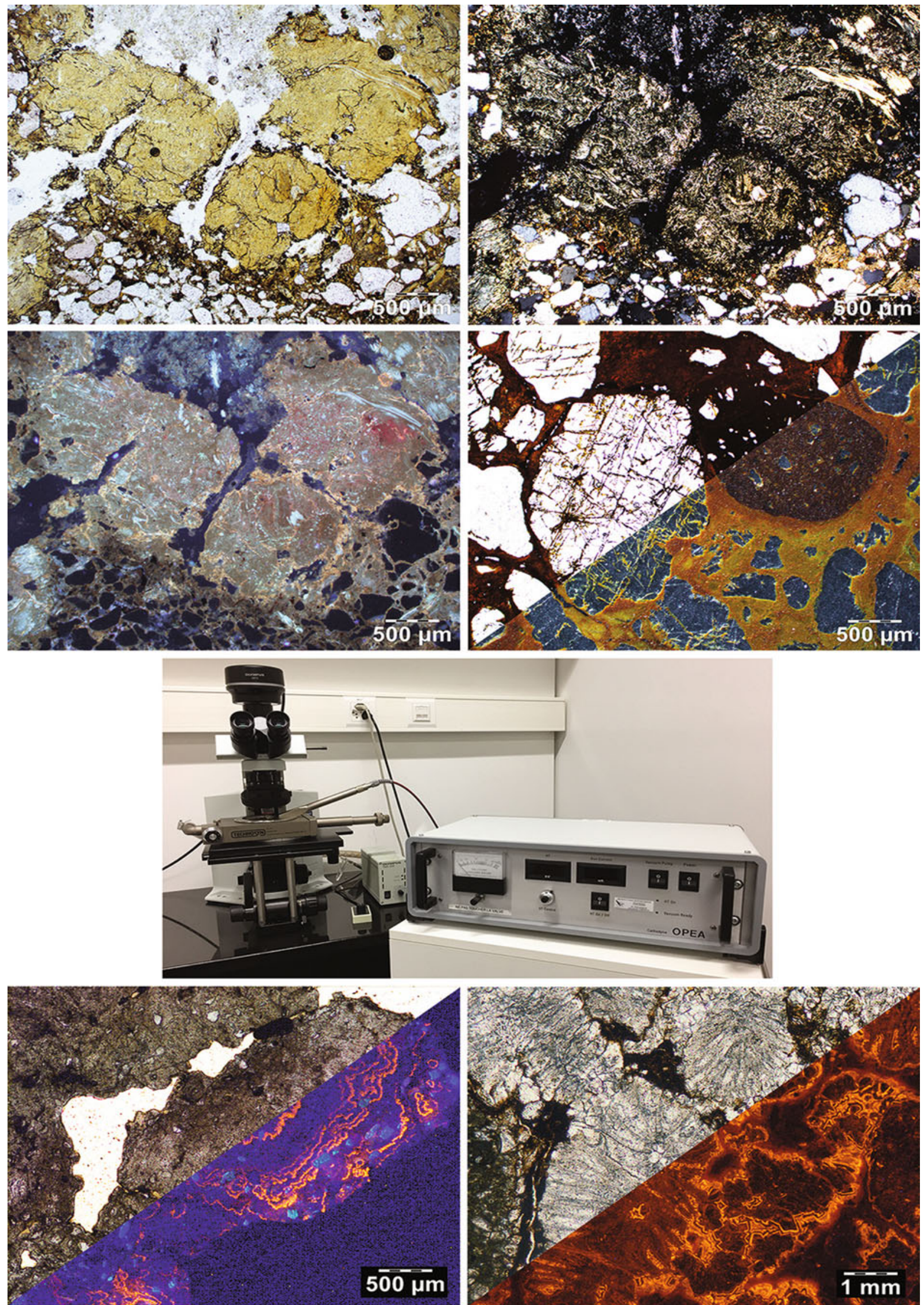


\section{File 6: Other Techniques of Observation}

In soil micromorphology, not only are observations made in transmitted light but incident light is also used. The two most common techniques refer to fluorescence and cathodoluminescence. The source of light in fluorescence is usually an intense high-pressure mercury lamp ranging from 50 to $250 \mathrm{~W}$. A high energy electron beam is the source of excitation in cathodoluminescence at a voltage between 10 to $20 \mathrm{kV}$. This equipment needs adaptation for the polarized light optical microscope.

\section{Captions from upper left corner to lower right corner.}

1. Yellow aggregates containing organic matter and phosphate from a dung deposit. Bottom part of the photograph: quartz sand in a dark and grey micromass. PPL.

2. Same view as 1. in XPL. Organic matter and phosphate remain extinct.

3. Same view as in items 1. and 2. under fluorescence blue-exciting radiation. The phosphate compounds appear in yellow, and the quartz grains are extinct (black). Some organic matter is excited and appears in bright pale blueish colours.

4. View in oblique incident light (OIL; lower right corner). OIL is a technique using direct reflected light projected on the thin section. It is widely used in archaeology (Goldberg and Macphail 2003; Nicosia and Stoops 2017). Dark greyish brown hematite-rich nodule surrounded by dark grey quartz in a micromass from a Ferralsol, Burkina Faso.

5. Optical microscope equipped with a cold-cathode cathodoluminescence (CL) device (University of Lausanne). A CL-stage, in which the sample is surrounded by an ionized gas in a moderate vacuum, is attached to the microscope. The electron beam used to bombard the thin section with high-energy electrons is generated by the discharge taking place between the cathode and the anode, at ground potential. The energy carried in the beam interacts with the crystal matter, resulting in the luminescence of minerals (Marshall 1988; Pagel et al. 2000; Boggs and Krinsley 2006).

6. Upper left corner: a carbonate nodule (PPL). Calcite appears in various brown tones with some small whitish quartz grains. Lower right corner: view in CL emphasizing multiple and alternating reddish to yellowish layers due to the presence of $\mathrm{Mn}^{2+}$ in the crystal lattice. The purple-blue luminescence is due to the intrinsic luminescence of $\mathrm{Mn}^{2+}$-poor calcite (Richter et al. 2003).

7. Upper left corner: Microcodium, an unexplained feature currently observed in Tertiary paleosols developed in floodplains (PPL). Lower right corner: view in CL showing the structure of Microcodium in dark orange. The light orange rims are related to late diagenetic recrystallization, the luminescence being bright at a moderately high $\mathrm{Mn}^{2+}$ content, if $\mathrm{Fe}^{2+}$ abundance is below about 2000 ppm (Boggs and Krinsley 2006). 

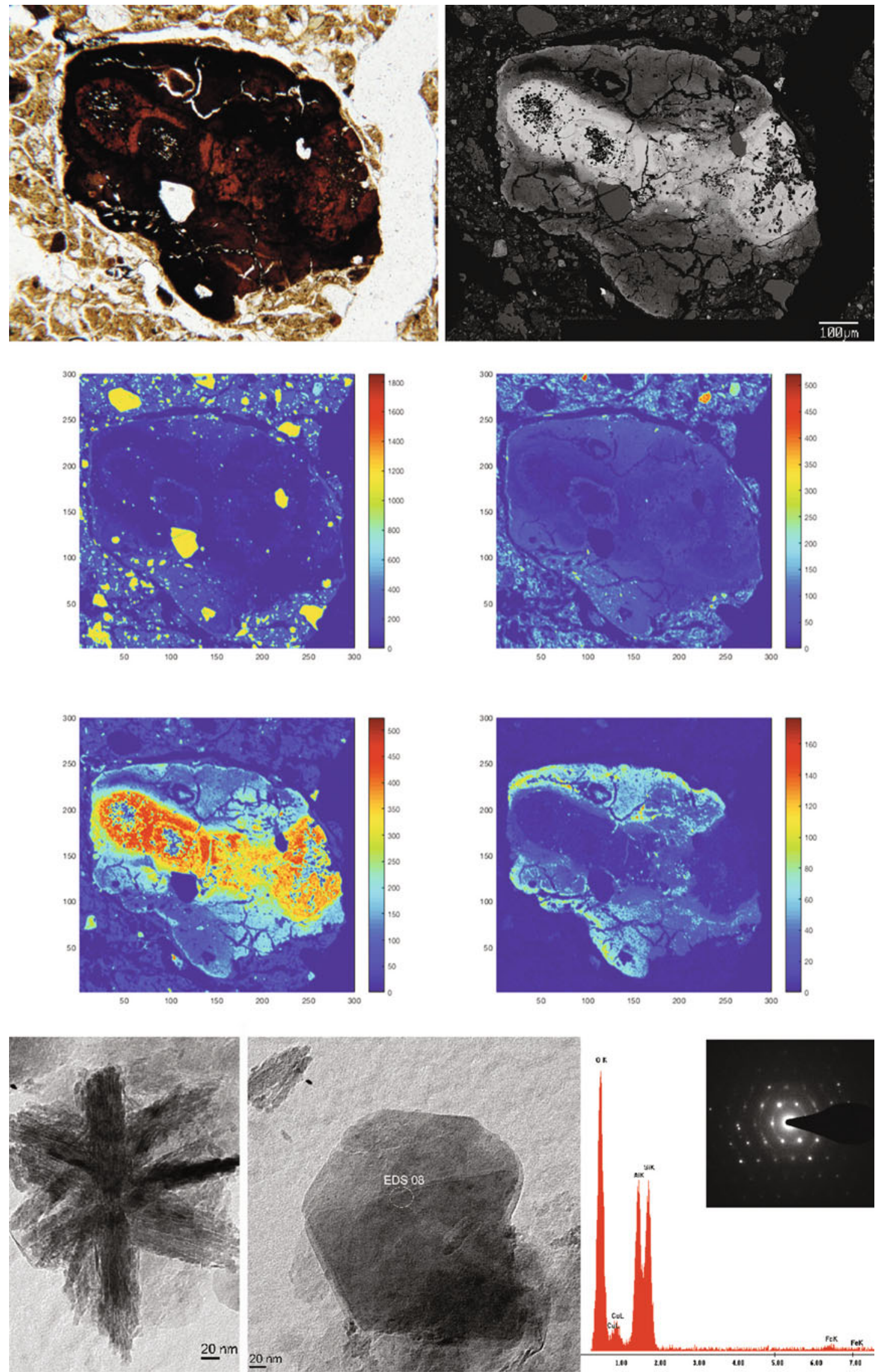


\section{File 7: Electron and Energy Imaging}

Soil micromorphologists can use the scanning electron microscope (SEM) to create images of features observed in thin sections. Not only can they access images at high resolutions, in order to see the minute structure of minerals and organic matter, but it is also possible to get information regarding the chemical composition of features. Electron probe micro-analyses (EMPA) are commonly performed to generate maps of chemical element distributions, whereas, transmission electron microscopes (TEM) are usually used to observe e.g. the structure of clay minerals or oxyhydroxides (see "File 78").

\section{Captions from upper left corner to lower right corner.}

1. Nodule composed of oxyhydroxides in a Cambisol developed on loess (Jura Mountains, Switzerland). The mineral fraction around the nodule includes some quartz grains and clay minerals (view in PPL).

2. Same nodule observed with an EMPA using the backscattered electron detector (BSE mode). The number of backscattered electrons reaching the detector is proportional to the mean atomic number of the sample: therefore, the light grey area correlates with heavier atoms, i.e. Fe and $\mathrm{Mn}$, whereas the darker areas denote lighter elements (e.g. $\mathrm{Al}$ or $\mathrm{Si}$ ).

3. Distribution map of Si: quartz grains appear in yellow or light green. They have high Si contents. The fine compounds around the nodule are enriched in Si compared to the nodule, which is highly depleted in $\mathrm{Si}$.

4. Distribution map of Al: this map emphasizes the clay content of the fine fraction (light blue and green) around the nodule. The combination of $\mathrm{Si}$ and $\mathrm{Al}$ confirms the presence of aluminosilicates, in this case phyllosilicates.

5. Distribution map of Fe: the nodule contains a very high proportion of $\mathrm{Fe}$ in its centre, with a decreasing gradient towards its border.

6. Distribution map of $\mathrm{Mn}$ : the nodule periphery is enriched in Mn, whereas its centre is extremely depleted, as well as the fine fraction of the soil.

7. TEM view of a hematite crystal observed in the same Cambisol.

8. TEM view of an inherited crystal of kaolinite showing its hexagonal shape. At this magnification, it is possible to observe some superposition of individual sheet crystals. The "EDS 08 " label refers to the spot analysed by EDS in 9.

9. In red, a spectrum of an EDS (Energy Dispersive X-ray Spectroscopy) analysis of the kaolinite crystal observed in 8. The spectrum shows the presence of $\mathrm{Al}, \mathrm{Si}$, and $\mathrm{O}$, as expected. The presence of $\mathrm{Cu}$ is due to some contribution from the grids on which the sample is deposited. In the right upper corner, an image of the beam's electron diffraction pattern obtained for the kaolinite crystal. In order to get such images, the sample is tilted using a goniometric stage attached to the TEM. 

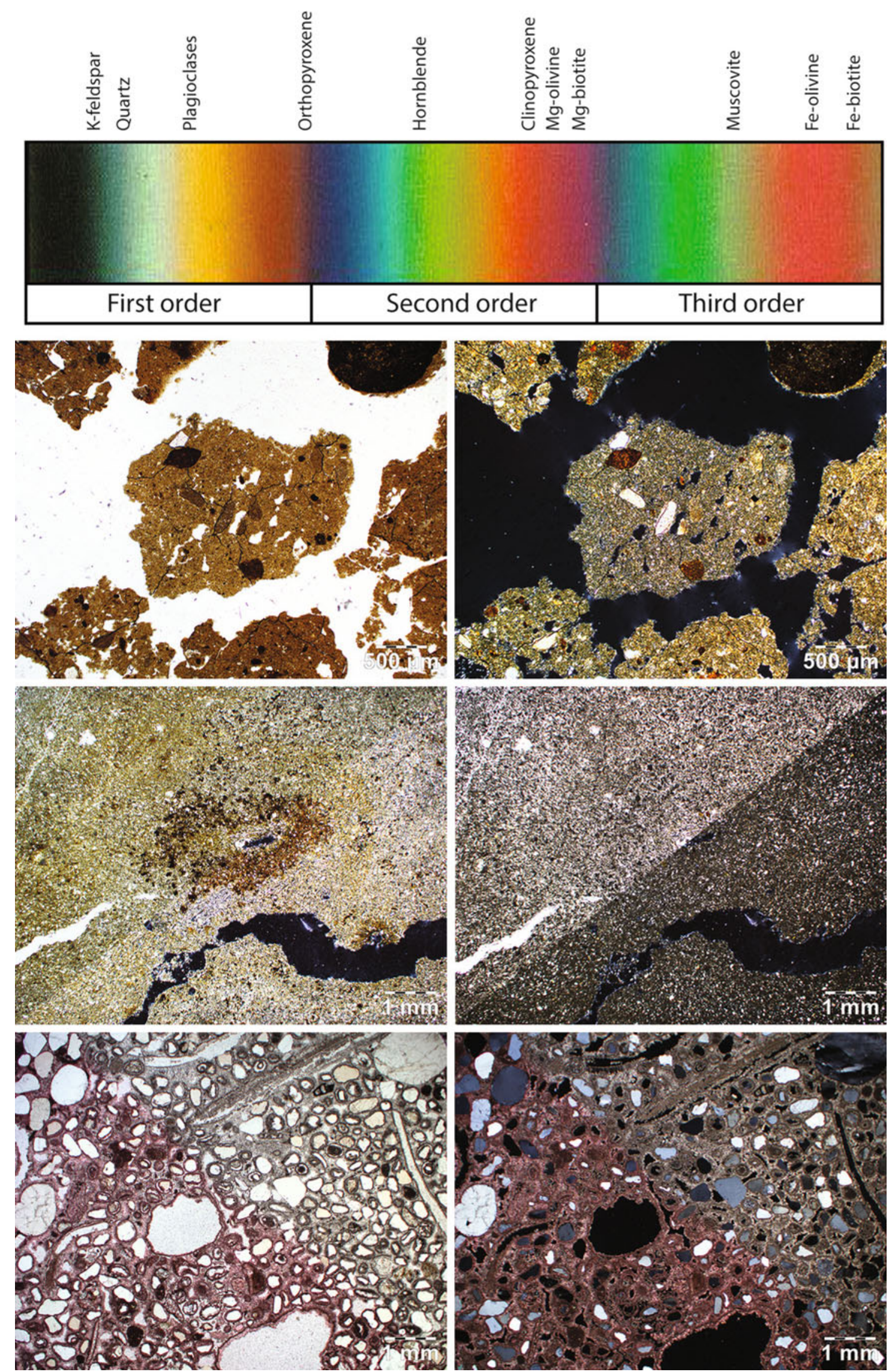


\section{File 8: Colours of Minerals}

When using a polarized microscope, light, which vibrates in a single plane, passes through the anisotropic minerals of the thin section (see "File 5"). Then, it splits into two beams perpendicular to each other, as it crosses the minerals, and propagates at different speeds according to the two refractive indices. The two vibrations emerge out of phase and pass through the analyser, which leads to the disappearance of certain wavelengths and to a resultant one, which defines the polarisation colour.

Captions from upper left corner to lower right corner.

1. In a $30 \mu \mathrm{m}$ thick thin section, mineral colours are a function of the difference between the maximum and minimum indices of refringence, a property called birefringence. It depends on the orientation of the crosspolarized plane with respect to the crystalline system of the mineral. The birefringence varies with the rotation of the stage, having four intensity maxima and four extinction positions (see "File 5"). The colour chart, taken from a Michel-Lévy table of birefringence, covers different orders, from left to right, according to increasing retardation. Retardation refers to the distance that the slow beam lags behind the fast one by the time that the slow beam finally exits the mineral. Common inherited minerals observed in soils are plotted alongside the chart in relation to their maximal polarisation colours of their highest birefringence.

2. View in PPL of aggregates. Pores, as well as some minerals, appear in white.

3. Same sample as in item 2. in XPL. In the pore void, there is no retardation, so no light passes through the analyser, this area staying extinct. When minerals are clear in PPL and extinct in XPL, regardless of the orientation of the stage, they are called isotropic. Organic matter is often isotropic. Conversely, some minerals can remain white in XPL, a first order colour due to slow retardation, whereas other grains shift from very dark brown to light orange.

4. Oxyhydroxide accumulation around a pore. There are also oxyhydroxide and calcium carbonate impregnations in the groundmass, giving a yellowish to brownish colour to the sample.

5. Same sample as in item 4. after selective extraction of $\mathrm{CaCO}_{3}, \mathrm{Fe}$, and $\mathrm{Mn}$. Such methods allow b-fabric, clay coatings, and microcrystalline features to be enhanced. It can also reveal the nature of the parent-material groundmass.

6. Recent soil developed on a siliclastic and carbonate sediment (PPL). The lower left triangle has been soaked in a solution containing alizarine red and potassium ferricyanide (Stoops 2003, 2021), staining high Mg-calcite red.

7. Same sample as in item 6. in XPL. Quartz grains appear in white, grey, and extinct, calcite in red (lower left triangle) or greyish brown (not stained; upper right triangle). 


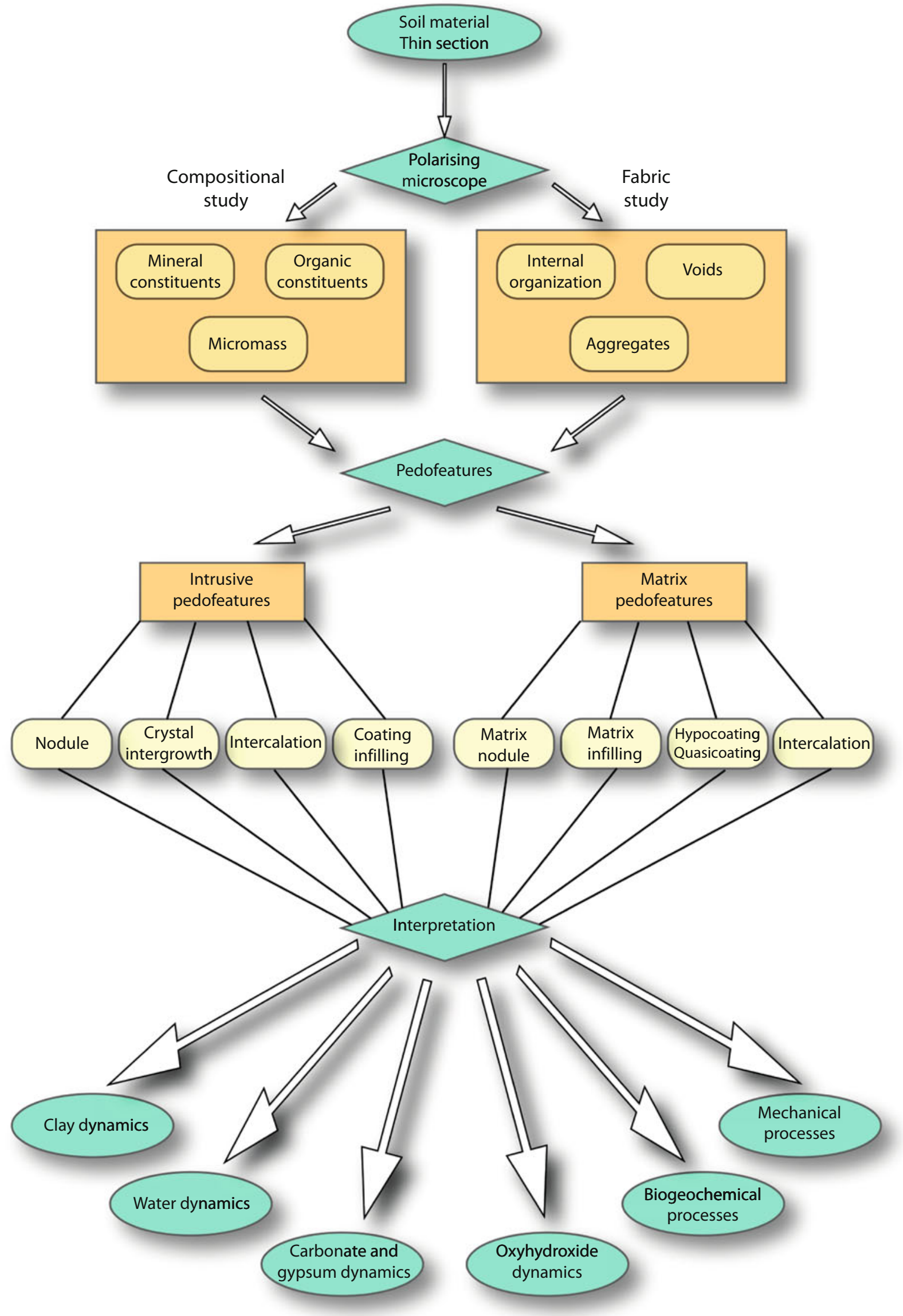


File 9: The Micromorphological Approach

The micromorphological approach is based on multiscalar observations of both composition and fabric. The chart is derived from the approach proposed by Stoops (2003). The oval boxes refer to input and output of the flow, the diamonds to decisions, rectangles to an identification process, and rounded rectangles to objects.

\section{Captions from top to bottom.}

1. The soil material is sampled in the field (see "File 3") and the thin section is prepared (see "File 4").

2. The study starts with the investigation of the groundmass using a polarizing microscope (see "File 5"). The groundmass is the base material of the soil in thin section. Two different branches of investigation are carried out to study this groundmass: (1) the compositional study (see Chap. 3) dealing with "data such as chemical and/or mineralogical composition or associated characteristics such as colour, refractive index, or interference colours" (Stoops 2003); the studied objects include mineral constituents, organic constituents, and micromass; and (2) the fabric (see Chap. 2), which focuses on the microstructure, aggregates, and voids.

3. After the descriptions of the various components and fabric of soil thin sections, the next step consists of the identification and description of pedofeatures (see Chap. 4). Pedofeatures are "discrete fabric units present in soil materials that are recognizable from adjacent material by a difference in concentration in one or more components or by a difference in internal fabric" (Bullock et al. 1985).

4. There are two main groups of pedofeatures: the matrix pedofeatures and the intrusive pedofeatures (Stoops 2003, 2021). The former refers to changes within the groundmass. The latter deals with changes outside the groundmass.

5. Each of the two groups of pedofeatures is subdivided into different subgroups, according to their morphology (Stoops 2003, 2021).

6. All the information gathered is used to interpret the thin section in terms of soil genesis, soil classification, soil use, palaeopedology, and geoarchaeology. However, the pedogenetic processes are generally not defined on a morphological basis, but mainly on chemical, physical, and biological properties and by using comparisons between profile horizons. Nonetheless, soil micromorphology is useful for the identification of the main dynamics affecting the soils.

7. Examples of six different dynamics that can be identified using soil micromorphology (see Chap. 5): the dynamics of clays, the impact of water, the precipitation of carbonates, sulphates, and chlorides, the formation of oxyhydroxides, the development of biogeochemical processes, e.g. organomineral interactions, and mechanical processes, e.g. gravity, compaction, or ploughing.

Open Access This chapter is licensed under the terms of the Creative Commons Attribution 4.0 International License (http:// creativecommons.org/licenses/by/4.0/), which permits use, sharing, adaptation, distribution, and reproduction in any medium or format, as long as you give appropriate credit to the original author(s) and the source, provide a link to the Creative Commons license, and indicate if changes were made.

The images or other third party material in this chapter are included in the chapter's Creative Commons license, unless indicated otherwise in a credit line to the material. If material is not included in the chapter's Creative Commons license and your intended use is not permitted by statutory regulation or exceeds the permitted use, you will need to obtain permission directly from the copyright holder.

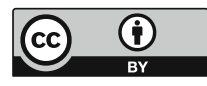

